\title{
A hierarchical-drift diffusion model of the roles of hunger, caloric density and valence in food selection
}

\author{
P. Garlasco ${ }^{\text {a }}$, S.A. Osimo ${ }^{\text {a }}$, R.I. Rumiati ${ }^{\mathrm{a}, \mathrm{b}}$, V. Parma ${ }^{\mathrm{a}, \mathrm{c}, *}$

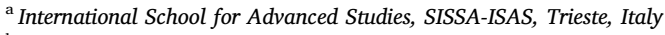 \\ ${ }^{\mathrm{b}}$ Agenzia Nazionale per la Valutazione dell'Università e della Ricerca (ANVUR), Roma, Italy \\ ${ }^{\mathrm{c}}$ William James Center for Research, Instituto Superior de Psicologia Aplicada (ISPA), Lisbon, Portugal
}

\section{A R T I C L E I N F O}

\section{Keywords:}

Decision-making

Food choice

Drift-diffusion model

Bayesian inference

Hunger

\begin{abstract}
A B S T R A C T
Decisions based on affectively relevant stimuli, such as food items, hardly follow strictly rational rules. Being hungry, the food's caloric density, and the subjective valence attributed to various foods are known factors that modulate food choices. Yet, how these factors relatively and altogether contribute to the food choice process is still unknown. In this study, we showed 16 healthy young adults low- and high-calorie food when hungry or fed, and we asked them to evaluate the valence of each visually-presented food. To compute the relative influence of hunger, caloric density and valence on food choice, we applied a hierarchical drift diffusion model (HDDM). Results indicated that hunger, caloric density and valence affected how fast participants accumulated information in favor of the chosen item over the other. When fed, participants were faster in choosing low-calorie foods and foods with a higher valence. Conversely, when hungry, participants were faster in choosing highcalorie foods, including food items with lower subjective valence. All in all, these findings confirm the complex nature of food choices and the usefulness of nuanced computational models to address the multifaceted nature of decision-making and value assessment processes affecting food selection.
\end{abstract}

\section{Introduction}

Food choice is a complex decision process (Rangel, 2013), influenced by several factors (Mela, 2001; Shepherd \& Raats, 2006; Shepherd \& Sparks, 1994). Some of these factors are food-related, such as the physical and chemical features of a food item (i.e., nutritional content, edibility; Wrangham, 2009). Some others are person-related, such as the physiological state of an individual who is called to make the choice (e.g., hunger, satiety, and thirst; Griffioen-Roose et al., 2014), sensory perceptual skills [i.e., how the food features are perceived subjectively with respect to texture, Foroni, Pergola, and Rumiati (2016)], body-mass index (Pergola, Foroni, Mengotti, Argiris, \& Rumiati, 2017), as well as transient [i.e., mood, stress, Zellner, Saito, and Gonzalez (2007)] and stable preferences [i.e., personality, Lindeman and Stark (1999)], cultural factors (e.g., eating turkey on Thanksgiving; Shepherd, 2001; Croker, Whitaker, Cooke, \& Wardle, 2009) and considerations about long-term effects of food choice on personal and societal well-being (e.g., obesity. See Shepherd, 2001; Stroebele \& De Castro, 2004; Toepel, Knebel, Hudry, le Coutre, \& Murray, 2009; Van der Laan, De Ridder, Viergever, \& Smeets, 2012).

Of these modulators, hunger and calories have been more extensively investigated. Hunger is the fundamental drive that motivates the search and consumption of nutrients. For instance, Piech, Pastorino, and Zald (2010) showed that the ability of food stimuli to capture attention and prevent target detection is increased when participants are hungry. When participants are satiated, and in particular when the motivational value for that stimulus has been specifically reduced (i.e., selective satiation), the attentional focus (as well as its subjective pleasantness) to a food item is limited.

Food calories have also been deemed a primary driver in food choices. In an evolutionary perspective, identifying high-calorie food represents a competitive advantage allowing to secure energy resources (Lieberman, 2014; Pontzer et al., 2016; Wrangham, 2009). Humans have evolved dedicated neural pathways and cognitive strategies to extract calorie information rapidly and reliably (Frank et al., 2010; Killgore et al., 2003; Toepel et al., 2009). When considering how calories are contributing to food choices, Charbonnier, Van Der Laan, Viergever, and Smeets (2015) found that satiated participants comparing equally likable food items chose overall more often and quickly low-than high-calorie foods. The authors contend that in lack of a physiological drive and when personal preferences are controlled for, choosing low-calorie food is adaptive to maintain a stable weight in the

\footnotetext{
* Corresponding author. International School for Advanced Studies, SISSA Via Bonomea, 265 34136, Trieste, TS, Italy.

E-mail addresses: vparma@sissa.it, vparma@ispa.pt (V. Parma).
} 
context of secure food availability (i.e., industrialized countries). In line with this interpretation, individuals eating with restraint (Gilhooly et al., 2007) or undergoing a fasting period (18hrs, Siep et al., 2009) tend to crave more high-than low-calorie foods (Gilhooly et al., 2007). Also, when asked to decide upon unhealthy (high-calorie) and healthy (low-calorie) food items, individuals in a state of hunger choose more high-calorie foods (Read \& Van Leeuwen, 1998). On the whole, hunger increases the salience of calorie-rich foods.

Another way of increasing the salience of particular food items, especially when food cues are ubiquitous, is to evaluate their subjective value (Spence, Okajima, Cheok, Petit, \& Michel, 2016). Hoefling and Strack (2010) show that satiated participants engaged in a forcedchoice binary food task tended to prefer items they liked more, irrespective of the time before consuming them. However, for hungry participants, food choice is driven by immediate availability of foods rather than by the valance attributed to food. Finlayson, King, and Blundell (2007) evaluated the effect of hunger, calories and valence on food choice. Their findings reveal that hungry participants chose more high-nutrient food items, with a selective modulation of the participant valence ratings on certain food categories (e.g., savory food). This study is in line with the argument that hunger, calorie and valence constitute a multi-determinant food choice mechanism. Taken together, these studies suggest the usefulness of multidimensional approaches to understand and predict food choices (Koster, 2003).

A paradigm often used to investigate food choices involves binary food choices (BFC). BFC tasks have been widely used in the behavioral economic and neuroeconomic literature (e.g., Camerer, Loewenstein, \& Rabin, 2011; Padoa-Schioppa \& Assad, 2006) to model complex choices in a computationally tractable, yet ecological manner. To account for multiple decision factors and rule out the non-relevant noisy information, we relied on Bayesian Causal Inference (Rohe \& Noppeney, 2015), which consists in estimating the probability of an occurrence based upon prior information and weighted by the current evidence. Choice models have often capitalized on a family of models called drift-diffusion models [DDM, Ratcliff (1978)]. In a Bayesian framework, such models allow the quantification of the speed of the decision process based on factors such as the accuracy (e.g., Cavanagh et al., 2011; Vandekerckhove, Tuerlinckx, \& Lee, 2011). Using as input the distribution of reaction times (RTs) and the accuracy of the responses, DDMs assume that the subject is processing the information by accumulating evidence for each of two alternatives (i.e., two food items) until a threshold for a decision (i.e., food choice) is met. Compared to classical frequentist analyses, these models allow the quantification of uncertainty based on the data and an informed prior distribution, instead of relying on a theoretical (usually Gaussian) distribution. Furthermore, they allow for simultaneous consideration of the speed of the accumulation of information (drift-rate, $v$ ) and the point at which the decision is made (threshold/boundary, a), a possible pre-existing bias towards one of the two options (bias, $\mathrm{z}$ ) and also non-decision related components of the task (e.g., perception, movement initiation and execution are all summarized in the parameter $t$ ). This approach favors the joint investigation of critical aspects of the choice, rather than separating them into different models, as the frequentist approach calls for. The reaction times (RTs) and the pattern of choices are therefore interpreted as evidence of the underlying cognitive processes, whose investigation was the scope of this study. The possibility of inferring different cognitive processes using a modeling approach is therefore an improvement over simpler RTs modeling. A linear mixed model approach, for instance, would not allow to differentiate between the time related to decision making and the motor response, or whether there is a bias towards a certain type of food (e.g., high-calorie food).

A handful of studies has employed DDM to study specifically food choices (e.g., Krajbich, Armel, \& Rangel, 2010; Mormann, Malmaud, Huth, Koch, \& Rangel, 2010; Mormann, Koch, \& Rangel, 2011; Towal, Mormann, \& Koch, 2013). In particular, Towal et al. (2013) investigated how the perceptual saliency and subjective food valence influence fixation patterns and food choice. Their results indicate that fixation patterns modulate choice primarily in accordance with the valence of the food item, but also based on its perceptual saliency, confirming a complex interplay between bottom-up and top-down factors underlying even the most basic (binary) food choices. So far, the DDM applied to BFC has mostly considered one or two factors influencing food choice.

In the present study we extended the investigation on the patterns of accumulation of information while making binary food choices by investigating at the same time the effects of hunger, caloric density, and valence. To simultaneously assess the role of hunger and valence in food choice, we evaluated how participants chose high- and low-calorie food items in relation to their satiety state (hungry/fed) and of the subjective valence attributed to the food (high/low). We modeled the choice towards low- and high-calorie food, fully counterbalanced across participants, irrespective of the valence individually assigned to each food. We predicted that hunger would affect the boundary position (a), in that it would take less time to reach the high-calorie boundary when participants were hungry compared to when they were satiated (or smaller distance from the drift starting point to the boundary for highcalorie foods as compared to the distance from the drift starting point to the boundary for low-calorie foods). This would mean reducing the amount of information necessary to make the choice when hungry, as compared to when satiated. With respect to the drift rate, we expected participants to choose more rapidly low-calorie foods when fed, as found in other studies run in industrialized countries (Charbonnier et al., 2015), irrespective of food valence. When hungry, faster accumulation of information is presumed for high-calorie food, even when a lower valence has been attributed to them. In other words, we expected the drift rate to be greater in hungry than fed participants. Considering that participants should not be able to guess where a low- or highcalorie food items will be presented across trials, we hypothesized that neither hunger nor valence would influence the bias term of the model (z). As a control check, we did not expect the non-decision parameter $t$ to vary between the models.

\section{Materials \& methods}

\subsection{Participants}

Sixteen healthy individuals $(8 \mathrm{~F})$ participated in the experiment. Participants were right-handed, within a normal weight range (see Table 1), with normal or corrected-to-normal vision, were omnivorous and had no dietary restrictions. Participants were screened for dysfunctional eating habits through the Restraint Scale questionnaire (Polivy, Herman, \& Howard, 1988, pp. 377-380), inquiring into participants' weight fluctuations and concerns over dieting. All participants had scored within the normal range. Participants also exhibited regular sleep patterns and were not sleep deprived, as assessed with the Pittsburgh Sleeping Quality Index (PSQI, Buysse, Reynolds, Monk, Berman, \& Kupfer, 1989; in its validated Italian version, Curcio et al., 2013). Controlling for these factors limits the possibility of extreme choice patterns due to factors not directly manipulated in the experiment. Each participant was compensated with 8 Euro per session, for a total of 16 Euro. Table 1 summarizes the sample features. One participant (ID 2) was removed from final analyses given that the RT distribution of her responses was significantly different from the right-skewed distribution retrieved for all other participants (see Supplementary materials,

Table 1

Participants' demographic and questionnaires' score. BMI, Body Mass Index; RS, Restraint Scale; PSQI, Pittsburgh Sleep Quality Index.

\begin{tabular}{lllll}
\hline Age & Education & BMI & RS & PSQI \\
\hline $24.5 \pm 2$ & $17.6 \pm 1$ & $22 \pm 2$ & $11 \pm 5$ & $10 \pm 3$ \\
\hline
\end{tabular}


section $3 b)$.

\subsection{Stimuli}

Forty images were selected from a validated database (FRIDa, Foroni, Pergola, Argiris, \& Rumiati, 2013). Stimuli were divided in two groups of interest based on their caloric density/100 g. In line with other studies (e.g., Ohla, Toepel, Le Coutre, \& Hudry, 2012) and with the nutrition database of the United States Department of Agriculture (www.nal.usda.gov/fnic) we decided to use $151 \mathrm{Kcal} / 100 \mathrm{~g}$ as a cut-off for low-calorie foods. In order to maximize the differences in caloric density between the two groups we also excluded high-calorie food that had less that $300 \mathrm{Kcal} / 100 \mathrm{~g}$. Out of the forty images, twenty were lowcalorie foods (range from 0 to $150 \mathrm{Kcal} / 100 \mathrm{~g}$ ) and twenty were highcalorie foods (range from 300 to $450 \mathrm{Kcal} / 100 \mathrm{~g}$ ). In each group, ten images of savory and ten images of sweet foods were presented to account for any sweet/savory preferences, in line with other studies (Mormann et al., 2010, see Supplementary Fig. S1 for a few examples). To reduce the confounding effect of known variables affecting the choice of a high- or low-calorie food, we selected images from the FRIDa database so that the norms for arousal, typicality, familiarity and valence did not significantly differ (Foroni et al., 2013). Portion sizes were those of standard servings of the food as available in the database. Given the task instructions (as reported in supplementary materials, section 2) we believed that the exact portion size would not be crucial in determining participants' choices. In line with other studies (Brunstrom \& Rogers, 2009), we did not expect portion size to affect the perceived valence of the foods, as it would have been the case if the instructions focused on consumption instead. To clarify how valence and hunger impact on the accumulation of information determining the choice of a low-vs. High-calorie food, we tested how this choice unfolds in four different choice options. We asked participants to choose between high-vs. High- (200 trials), low-vs. low- (200 trials), high-vs. lowand low-vs. High-calorie food items (400 trials, depending on the position of presentation of the food items in the pairs). This was done to evaluate whether choosing, for instance, a low-calorie food in the presence of another low-calorie food would be different than when it is chosen in the presence of a high-calorie food. Since no significant differences in the accumulation of information was retrieved due to the choice being made in a same vs. different caloric context (linear mixed model with RTstd $\sim$ session $\times$ valence * calorie $\times$ condition, $t=1.74$, $\mathrm{p}=0.08$ ), we collapsed data across conditions, and we used the calorie as a boundary for the HDDM decision. The total number of trials per session $(\mathrm{N}=800)$ was in line with previous studies (Mormann et al., 2010), and was meant to provide enough data points for the DDM to converge. The stimuli were pseudo-randomized so that a stimulus was never compared with itself and it was presented an equal number of times in both locations (i.e., right vs. left choice). Half of the comparisons included a sweet item and the remaining half a savory item. Since the significant difference when comparing sweet and savory food items was not significant (linear mixed model with RTstd $\sim$ session $\times$ valence * calorie $\times$ sweet/savory, $t=-0.85, \mathrm{p}=0.40$ ), this comparison was not included in further analyses.

\subsection{Experimental procedure}

Each participant came to the lab for two experimental sessions, taking place at the same time (to the hour), three days apart from each other. Participants were instructed to fast (only water allowed) for the $12 \mathrm{~h}$ prior to the beginning of the experiment. In one session ('hungry' condition), participants had to perform the task without eating, while in the other session ('fed' condition), they were offered cereal bars to eat up to satiety before performing the task. The order of the sessions was counterbalanced across participants. Afterwards, they filled in the questionnaires on sleeping habits (PSQI) and rated their hunger and thirst level and their tiredness on a 10-point scale. Fasting time in the

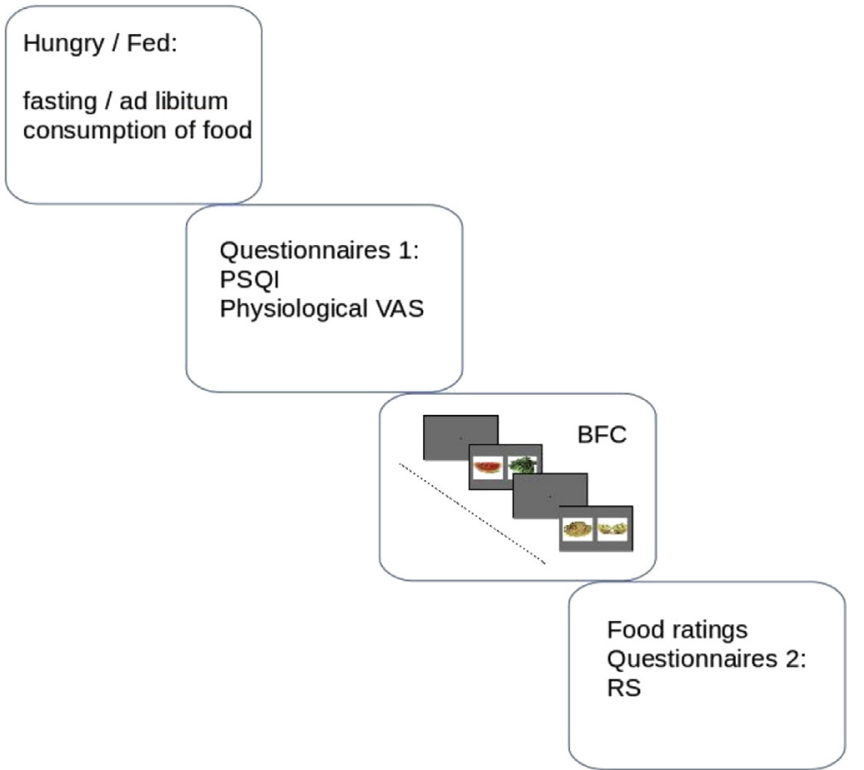

Fig. 1. The timeline of the procedure of the experiment for each session (fasting/fed).

literature ranges from 3hrs (Frank et al., 2010) to 18hrs (Siep et al., 2009), with behavioral effects emerging already at its lowest end (i.e., $3 \mathrm{hrs}$ ). The ratings confirmed that our manipulation between sessions was effective (Wilcoxon signed-rank test: hunger, $\mathrm{V}=120, \mathrm{p}<0.001$; thirst, $\mathrm{V}=31.5, \mathrm{p}=0.31$; tiredness, $\mathrm{V}=48.5, \mathrm{p}=0.18$ ). Subsequently, participants performed the BFC task sitting in front of a computer (instructions are reported in section 2 of the Supplementary materials). Stimuli were presented on an LCD screen $(60 \mathrm{~Hz})$ located approximately $80 \mathrm{~cm}$ from the participant's median line using PsychoPy2 (Peirce, 2007). Participants were shown pairs of food images and they were instructed to quickly choose whichever food item they preferred (see Fig. 1), pressing either the " $z$ " key to choose the food on the left or the " $m$ " key to choose the food on the right (for full instructions please refer to the Supplementary material). These keys were chosen to allow enough physical space between the right and the left buttons not to be confused.

Before each trial, participants had to fixate a cross for a random interval between 1 and $2 \mathrm{~s}$. To facilitate the investigation of spontaneous food choices, participants had a maximum of $3 \mathrm{~s}$ to make their choices (e.g., Mormann et al., 2010). If 3 s elapsed without a key press, the response was considered null, and the pair was not presented again. Null responses amount to $1 \%$ of trials (equally distributed across conditions). The task lasted approximately $40 \mathrm{~min}$, and participants were allowed to take three breaks to prevent fatigue ( $\max 1 \mathrm{~min}$ ). After the task, participants rated the valence of each food image from 1 to 10 , one indicating a very low valence, and 10 a very high valence. To make this variable computationally tractable, we reduced this continuous variable to a categorical one. Ratings from 1 to 4 were categorized as low-valence whereas ratings from 6 to 10 were categorized as highvalence. The food items that were rated 5 by participants were not included in the analyses in a subject-by-subject manner, since no clear valence could be attributed. Food valence was rated a posteriori, to avoid influencing participant's choice based on their expressed subjective food valence (e.g., Coppin et al., 2014). Afterwards participants filled in the RS questionnaire. The total time of a session lasted approximately $1 \mathrm{~h}-1 \mathrm{~h} 15$ '.

\subsection{Data analysis}

\subsubsection{DDM analysis}

The analysis was performed using a modified Bayesian version of a 
drift-diffusion model: the Hierarchical Drift Diffusion Model (HDDM). A HDDM employs Bayesian estimation of the model parameters providing a quantification of the reliability of such parameters (Vandekerckhove et al., 2011). Hierarchical Bayesian estimation permits constraining subject variability to a group-level distribution, so that individual differences can be minimized (Lee \& Wagenmakers, 2014; Regenbogen, Johansson, Andersson, Olsson, \& Lundstrom, 2016). Also, to control for noise, HDDM uses analytic integration of the likelihood function for variability in drift-rate and numerical integration for variability in nondecision time and bias (Ratcliff \& Tuerlinckx, 2002). As in Wiecki, Sofer, \& Frank, 2013, we do not attempt to estimate individual acrosstrial variability parameters. Adding these parameters made the models intractable and the payoff, i.e. a nuanced understanding of error responses in the RT distribution, was not crucial to our goals. In line with Wagenmakers, Van Der Maas, \& Grasman, 2007, we therefore decided to focus on the most psychologically relevant parameters (drift-rate, boundary, bias and non-decision time). Specifically, the models we tested were the following: i) valence $\times$ hunger: hddm. $\operatorname{HDDM}$ (data, p_outlier $=0.05, \backslash$ depends_on $=\{$ 'v': ['session', 'val_bin'], 'a': ['session', 'val_bin'], 't': ['session', 'val_bin']\}, bias = True); ii) valence: hddm. $\operatorname{HDDM}$ (data1, p_outlier $=0.05$, \ depends_on $=\{$ 'v': 'val_bin', 'a': 'val_bin', 't': 'val_bin'\}, bias = True); iii) hunger: hddm. HDDM(data1, p_outlier $=0.05$, $\backslash$ depends_on $=\{$ ' $v$ ': 'session', 'a': 'session', ' $t$ ': 'session'\}, bias $=$ True).

2.4.1.1. Model fitting. Data analysis was performed using the HDDM module for Python3 (Wiecki et al., 2013). To test whether choosing a low-calorie food in the same calorie context (i.e., paired with another low-calorie food) induced a differential drift than when choosing the same food item when paired with a different calorie food (i.e., highcalorie food), we run a HDDM with the four separate conditions, namely high-high, low-low, low-high and high-low. Given that this model was not able to converge, we collapsed the data across conditions. In other words, whenever a low-calorie food was chosen, that trial was used to model how the accumulation of information occurs for the choice towards the low-calorie boundary irrespective of the calorie context in which that low-calorie food is included (i.e., lowlow, low-high and high-low). Similarly, whenever a high-calorie food was chosen, those trials contributed to the accumulation of information necessary to reach the choice of a high-calorie food. In the model reported, we assumed that the slope of the drift of the choices of low- or high-calorie food would differ when such choices were performed under different levels of hunger and based on the valence of each food item. Each choice is represented as an upper boundary - here associated with high-calorie foods (in the figures represented on the right side of the $\mathrm{x}$ axis, with positive drift-rate values) - and a lower boundary - here associated with low-calorie foods (in the figures represented on the left side of the $\mathrm{x}$ axis, with negative drift-rate values). The bias, instead, indicated the starting distance from each of the two boundaries. Hence, we estimated the model a-posteriori distribution of the parameters by using Monte-Carlo Markov Chain simulation (MCMC) with gradient ascent optimization, drawing 10000 samples and burning the first 1000 to stabilize the model (Regenbogen et al., 2016). To allow for convergence analyses, we repeated the simulations 5 times per model (Wiecki et al., 2013).

2.4.1.2. Model convergence. Convergence of the models was inspected both visually and quantitatively. We plotted the trace of the models, the auto-correlation and the mean and distribution of the boundary, driftrate, bias and non-decision time (movement) parameters. We used the Gelman-Rubin $\mathrm{R}^{\wedge}$ statistic as a numerical estimate of the convergence (Gelman \& Rubin, 1992). $\mathrm{R}^{\wedge}$ values close to 1 indicate a small difference among the estimates of the different distribution of samples, which can be considered an index of the reliability of the simulations.

2.4.1.3. Model testing and comparison. To assess the reliability of the parameter estimates, we calculated the difference in mean probabilities of the posterior estimates of the conditions of interest (as done in Wiecki et al., 2013; Cavanagh et al., 2011). We report the data as probabilities transformed in percentages. Moreover, null hypothesis significant testing was performed with a rmANOVA with two withinsubject factors (hunger and valence) and two levels each (high and low). In case of significance, Bonferroni-corrected post-hoc tests were performed accordingly (as in Regenbogen et al., 2016). Model comparison using the Deviance Information Criterion (DIC), which is particularly suited for hierarchical models, determined the best model based both on goodness of fit and model complexity (Spiegelhalter, Best, Carlin, \& Van Der Linde, 2002). In this specific case, DIC allowed us to compare models including different numbers of parameters (e.g., only hunger vs. Hunger and valence), holding into account the complexity of the model itself, as a penalizing factor. With this criterion, lower values indicate a better fit of the model. Importantly, the values of DIC make sense only relative to each other, so it is meaningless to compare them with DICs from other studies (Gelman, Carlin, Stern, \& Rubin, 2014; Spiegelhalter et al., 2002).

\section{Results}

\subsection{HDDM}

\subsubsection{Model convergence and comparison}

Overall, our models converged satisfactorily. The auto-correlation of the last hundred trials (Fig. 2) was close to zero, as expected from a convergent Markov-Chain (Wiecki et al., 2013). This means that the samples are independent draws from the posterior. In Fig. 2, panel A, the trace is plotted as a function of the number of iterations. Moreover, the iterations do not stray too far away from the mean of the distribution (which is the point of highest probability of the posterior). The histogram on Fig. 2, panel C confirms this.

Moreover, the Gelman-Rubin statistics $\left(\mathrm{R}^{\wedge}\right)$ for our models was mostly close to 1 , (mean $=1.0002, \mathrm{SD}=0.0004$, for a total of 164 nodes per model) allowing to infer that different simulations of the same model obtained similar results, which is considered an index of robustness of the estimation itself. Overall, estimated non-decision time (parameter $\mathrm{t}$ ) lasted approximately $0.43 \mathrm{~s}$ and differed slightly among conditions [On-way repeated measures ANOVA with two factors (Hunger, Valence) and two levels (High, Low). Hunger: F $(1,1)=55.23$, $\mathrm{p}<0.001$; Valence: $\mathrm{F}(1,1)=19.96, \mathrm{p}<0.001$; Interaction: $\mathrm{F}$ $(1,1)=1.83, p=0.18]$. However, the difference was very small and hardly relatable to differences in underlying psychological processes (Table 2). This result is consistent with our data suggesting that given an average RT of $0.78 \mathrm{~s}$, the participants took approximately $0.45 \mathrm{~s}$ to decide on most images what to choose (the raw RT data are reported in the supplementary materials, section 3). Model comparison included all the three models we run (Hunger $\times$ Valence, Hunger, Valence). As reported in Table 3, the model with Hunger $\times$ Valence was the most reliable model, whereas the hunger model was the one with the highest DIC and thus the least reliable. This indicates that both hunger and valence contribute to the choice of the foods selected.

\subsubsection{Model results}

3.1.2.1. Boundary. The effect of Hunger, Valence or both factors together on the boundary parameter was close to chance level (50-52\%, see Table 4). A rmANOVA with the two within-factors (Hunger and Valence) and two levels (High and Low) was significant [Hunger: $\mathrm{F}(1,1)=25.38, \mathrm{p}<0.001$; Valence: $\mathrm{F}(1,1)=36.6$, $\mathrm{p}<0.001$; Interaction: $\mathrm{F}(1,1)=164.96, \mathrm{p}<0.001$ ] . However, as you can see from the posterior estimates (Fig. 3 and the probabilities reported in Table 3) the magnitude seems negligible. Therefore, the decision threshold for the high and low-calorie food can be considered equidistant. 

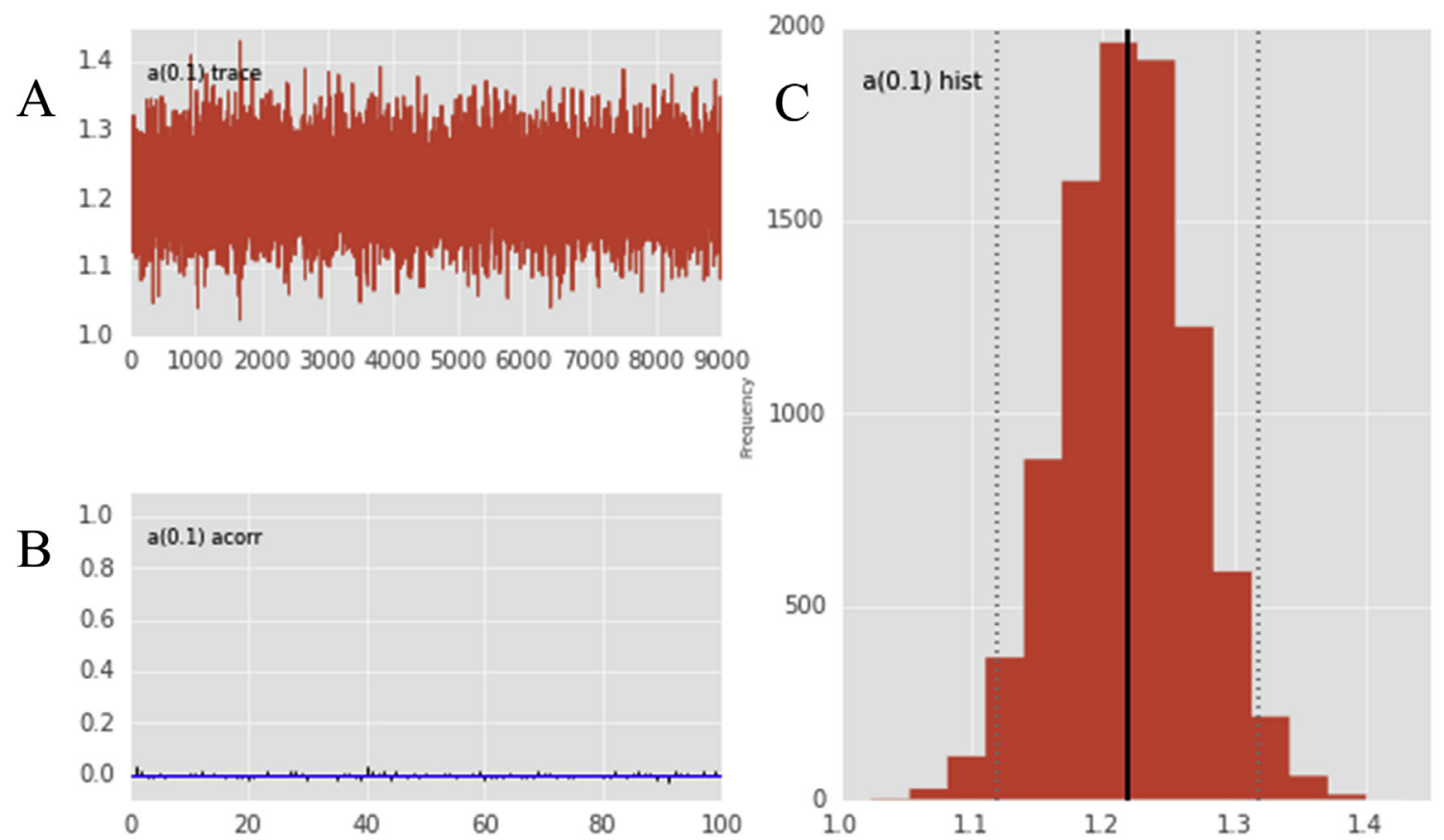

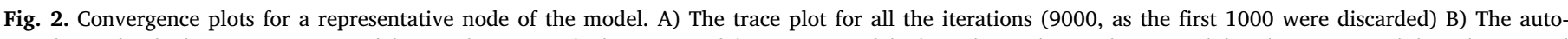

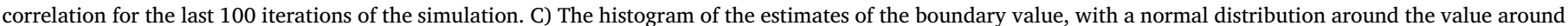
which the algorithm converged (1.22). The histogram shows the frequency of the estimates for each iteration of the algorithm.

Table 2

Mean and SDs by condition for the estimates of non-decision time (t) parameter.

\begin{tabular}{lllll}
\hline Condition & $\begin{array}{l}\text { Fasting - High } \\
\text { Valence }\end{array}$ & $\begin{array}{l}\text { Fasting - Low } \\
\text { Valence }\end{array}$ & $\begin{array}{l}\text { Fed - High } \\
\text { Valence }\end{array}$ & $\begin{array}{l}\text { Fed - Low } \\
\text { Valence }\end{array}$ \\
\hline mean & 0.438 & 0.435 & 0.433 & 0.431 \\
SD & 0.018 & 0.018 & 0.017 & 0.017 \\
\hline
\end{tabular}

Table 3

DIC values for our three HDDM. Lowest values represent a better fit. valence $\times$ hunger $=$ model with hunger and valence; valence $=$ model with valence; hunger $=$ model with hunger.

\begin{tabular}{ll}
\hline Model & DIC \\
\hline Hunger $\times$ Valence & 22710 \\
Valence & 24029 \\
Hunger & 25078 \\
\hline
\end{tabular}

Table 4

Valence $\times$ Hunger model. Probability difference of posterior estimates for boundary (a).

\begin{tabular}{lll}
\hline & Contrast & Probability (\%) \\
\hline \multirow{2}{*}{ Valence } & P(Fed, High Valence > Fed, Low Pref) & $51 \%$ \\
& P(Hungry, Low Valence > Hungry, High & $52 \%$ \\
Hunger & Valence) & \\
& P(Hungry, Low Valence > Fed, Low Pref) & $51 \%$ \\
& P(Fed, High Valence > Hungry, High & $52 \%$ \\
Hunger $\times$ Valence & Valence) & $51 \%$ \\
& P(Hungry, Low Valence > Fed, High & \\
& Valence) & $51 \%$ \\
& P(Fed, Low Valence > Hungry, High & \\
& Valence) & \\
\hline
\end{tabular}

3.1.2.2. Drift-rate. We found a main effect of hunger on the drift-rate. As it can be seen in Fig. 4a, participants chose more often low-calorie foods and were overall faster in choosing them when fed as compared to

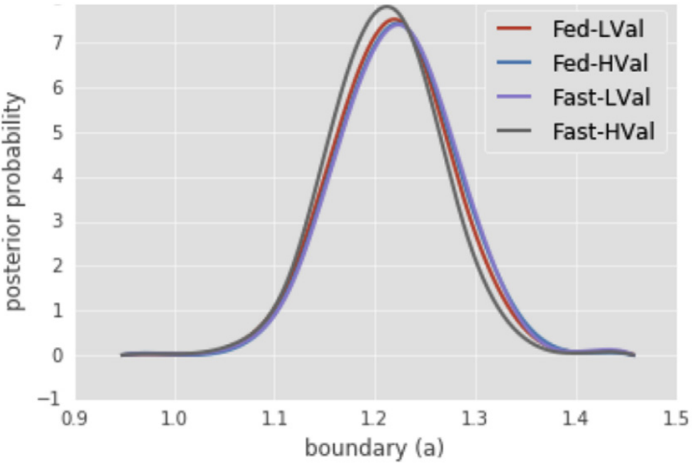

Fig. 3. Posterior probability distribution of the boundary (a) parameters. Valence $\times$ Hunger model with hunger $\mathrm{x}$ valence.

when they were hungry, as it is evident from the more negative drift for the fed session (95\%). Even preference alone had an effect on the driftrate, as displayed in Fig. 4b. Low-calorie food items seemed to be chosen faster when they were highly preferred (more negative drift), while more slowly when they were less preferred $(79 \%)$. In the Hunger $\times$ Valence model, we could find both main effects of Hunger and Valence, as well as their interaction (Table 5, Fig. 4c). A rmANOVA with the two within-factors (Hunger and Valence) and two levels (high and low) was in fact significant [Hunger: $\mathrm{F}(1,1)=19267$. $\mathrm{p}<0.001$; Valence: $\mathrm{F}(1,1)=25238, \mathrm{p}<0.01$; Interaction: $\mathrm{F}(1,1)=1.42$, $\mathrm{p}=0.23$. A six post-hoc Tukey test showed that both Hunger and Valence had a significant effect (Hunger: $t=-98.99, \mathrm{p}<0.001$, Bonferroni corrected; Valence: $\mathrm{t}=107, \mathrm{p}<0.001$, Bonferroni corrected). Different sessions seemed to impact on the speed of information processing as well as on the direction of the choice. Decisions taken when fed were overall faster towards low-calorie foods with a high probability, $98 \%$ and $80 \%$, for high and low valence, respectively. Interestingly a different pattern was observed when hungry. In this session, participants chose overall more highcalorie items when they had a low preference (70\%), while High Valence items tended to move the decision towards the low-calorie 

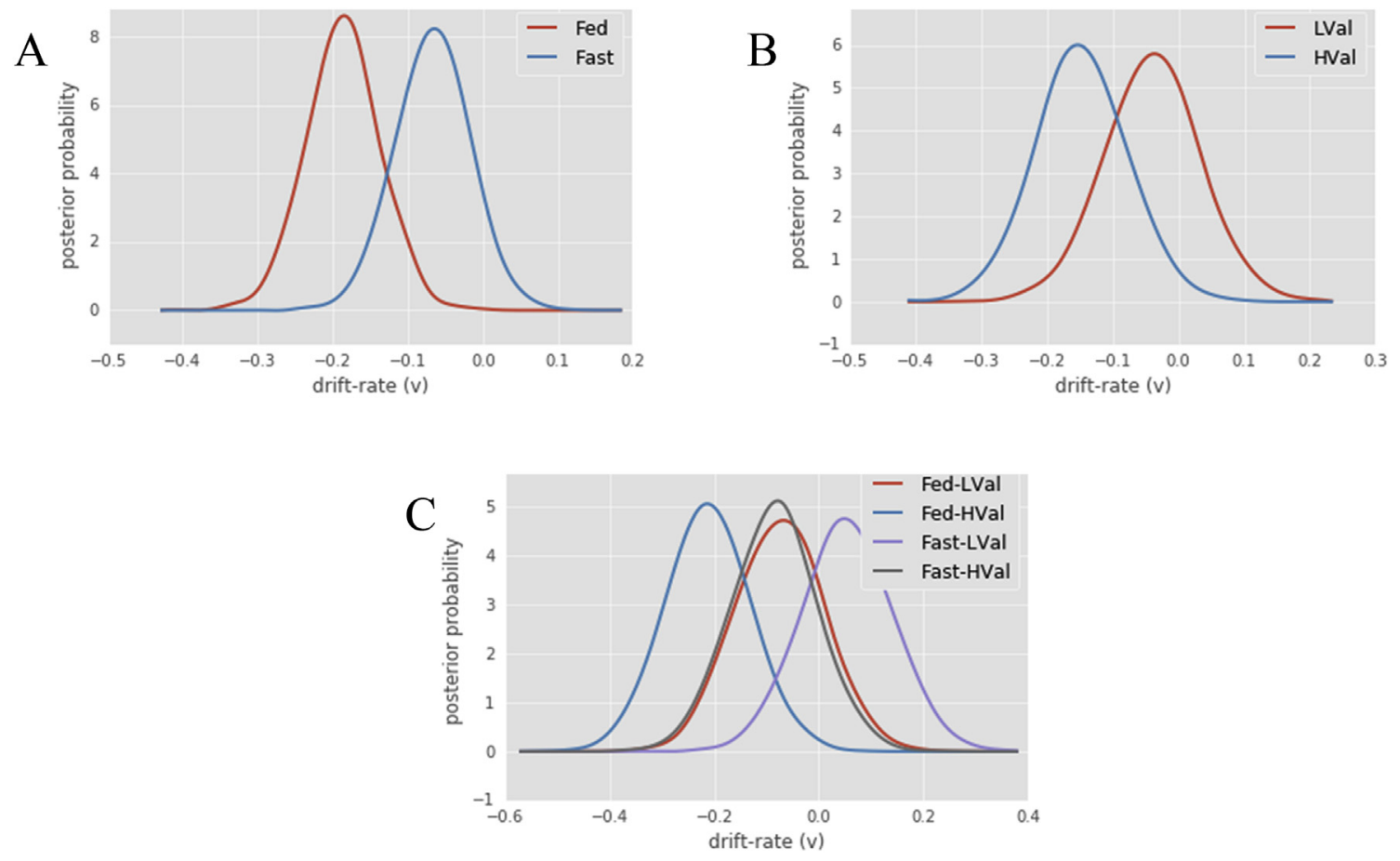

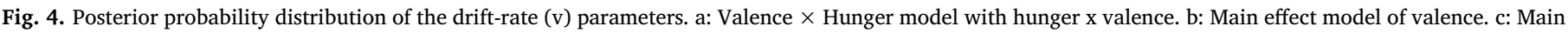
effect model of hunger. LVal = Low Valence, HVal = High Valence; Fast = Fasting.

Table 5

Valence $\times$ Hunger model. Probability difference of posterior estimates for driftrate (v).

\begin{tabular}{lll}
\hline & Contrast & Probability (\%) \\
\hline \multirow{2}{*}{ Valence } & P(Fed, Low Valence > Fed, High Valence) & $86 \%$ \\
& P(Hungry, Low Valence > Hungry, High & $80 \%$ \\
\multirow{5}{*}{ Hunger } & Valence) & \\
\multirow{5}{*}{ Interactions } & P(Hungry, Low Valence > Fed, Low Pref) & $83 \%$ \\
& P(Hungry, High Valence > Fed, High Valence) & $88 \%$ \\
& P(Hungry, Low Valence > Fed, High Valence) & $98 \%$ \\
& P(Hungry, High Valence > Fed, Low Pref) & $54 \%$ \\
& P(Fed, High Valence < 0) & $98 \%$ \\
& P(Hungry, Low Valence > 0) & $70 \%$ \\
& P(Hungry, High Valence < 0) & $79 \%$ \\
\hline
\end{tabular}

foods (79\%). Interestingly, the interaction between hunger and valence (98\%) sped up the accumulation of information in different directions. Towards low-calorie foods with high valence for the food and being fed and towards high-calorie foods with low valance and being hungry.

3.1.2.3. Bias. As expected, given the counterbalancing strategy chosen, the participants could not guess the location of either the low- or highcalorie food items across trials. Therefore, no bias towards either boundary emerged in any model (Fig. 5).

\section{Discussion}

Food choice is a complex decision that requires the assessment of conflicting information (Rangel, 2013). In this study, we investigated food choice by addressing the interplay of physiological and subjective factors. Using a DDM approach, we assessed how hunger, calorie and valence shape the patterns of accumulation of information in food choice. Having participants fast for $12 \mathrm{~h}$ successfully induced a state of hunger evident in the subjective ratings and the speed of accumulation of information necessary to reach the choice. Conversely, subjective valence for the food items was not modulated by the hunger state.

The results reported here show that choosing high-or low-calorie

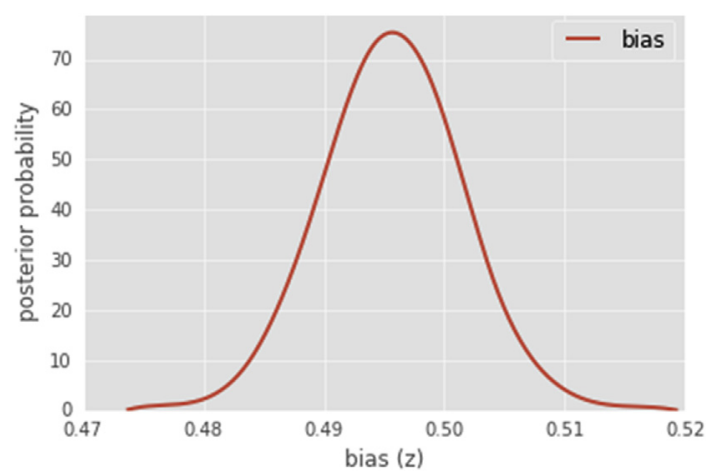

Fig. 5. Posterior probability distribution of the bias (z) parameter for the hunger $\times$ valence model.

food is equally probable, given that the boundary is equidistant from the starting point and that there is no previous bias towards either choice. In contrast with previous literature, suggesting that hunger makes less preferred high-calorie food more salient (Piech et al., 2010, di Pellegrino, Magarelli, \& Mengarelli, 2010), this result might be attributed to the preference for the food and lack of incentive in directly consuming the chosen food after the choice was made. The analysis of the posterior parameter distributions of the drift-rates instead showed significant differences in the speed of the decision process towards the two decision boundaries (low- and high-calorie food). In other words, as evident by the hunger $\times$ valence model, the speed was higher towards low-calorie foods when participants were fed, and the items had higher valence, while it was higher towards high-calorie foods when participants were hungry, and the foods had lower valence. This suggests that information used for food choices was accumulated faster for low-calorie, high-valence foods in the fed state than in the hungry state, whereas, when hungry, accumulation of information was faster for high-calorie foods, even if they were of low valence.

Here, we demonstrate that there is a complex interplay of factors in food choice with effects going in different directions - i.e., hunger, 
calorie and valence - which the HDDM approach allows us to disentangle and to point to specific aspects of the decision process. In line with Charbonnier et al. (2015), our participants chose more often lowthan high-calorie foods. The authors hypothesized that this was due to the fed state of their participants, which we confirm here by looking at the differences in the accumulation of information patterns for low-and high-calorie foods when fed, but not when hungry. Considering that overall there was no initial bias towards either food category based on calories, it can be argued that the physiological state of hunger is responsible for this food choice pattern. At the neural level, as suggested by Siep et al. (2009), these different patterns of accumulation of information may be underlined by a greater activation in the right insula and medial orbitofrontal and ventromedial prefrontal cortex for highcalorie foods when hungry. This would be in line with the role of orbitofrontal cortex in representing value-based decision alternatives, as previously contended (see Levy \& Glimcher, 2011). Finlayson et al. (2007) considered hunger, caloric value (fat content) and valence together. One of their main results is that hunger unbalanced the choices of participants towards highly fat foods (which can be equated to the high-calorie food items in our design). On the other hand, while satiated they were driven towards their preferred food irrespective of calories. In contrast with their results, in our study, hunger did not seem to affect our valence ratings, which were collected at the end of the session. Although we cannot exclude the possibility of an ownership bias (Frey, 1986) - the rating of the valence of the foods based on the choices made - we preferred to ask participants to rate the valence after the choice task not to induce false choices. Additionally, not presenting the food chosen to eat may also have partly flattened the hunger effect on the valence ratings. In spite of this, in our sample the analysis of the posterior drift-rates shows an effect of valence on the choice of low- and high-calorie foods. Additionally, this discrepancy in the subjective ratings may be due to a methodological reason. When controlling for arousal, typicality, familiarity and valence of the food images, the preference range may be constrained, therefore reducing variability irrespective of hunger states (Foroni et al., 2013). Furthermore, in line with Bielser, Cr'ez'e, Murray, and Toepel (2016), our data suggest that liked foods tend to be chosen more often than non-liked food, even though such pattern is not extremely skewed, due to a general preference for the food items presented. Such intuitive result confirms that liked items require lower decision times to be chosen (Kahnt, Park, Haynes, \& Tobler, 2014), even when the choice is forced among two alternatives (Bielser et al., 2016).

Overall, the present findings extend the investigation of food choice to the simultaneous evaluation of the effect of hunger, calorie and valence. This represents an improvement with respect to previous attempts which have been mostly focused on the analysis of self-report questionnaires or the evaluation of behavioral data with a frequentist approach, which limits the evaluation to one factor at a time. Conversely, applying a computational HDDM approach by simultaneously considering the effect of hunger, calorie and valence on food choice, allows us to overcome this shortcoming. Our results show that the food choice is not uniquely determined by fixed - i.e. exogenous preferences, but they demonstrate that real food choices are malleable to changes in physiological and subjective states, that is variables that can lie within the decision-process itself. Hereby, we contend that this approach is a strong candidate for the assessment of the complexities of food choice, contributing to a nuanced view of value-based decision in a unified framework (see Krajbich, Hare, Bartling, Morishima, \& Fehr, 2015). The importance of being able to experimentally model nuanced food value-based decisions seems to be reflected at the neural level by greater activation in a network of regions known to process saliencerelated information and cognitive control processes (Menon \& Uddin, 2010; Mitchell, 2011), including the insula, the dorsolateral prefrontal cortex, and the ventromedial prefrontal cortex.

One might argue that our data are based on fictional food choices since they were mediated only by food items that people visually inspected but were never able to eat. If on the one hand this represents a limitation of the current study, it extends a literature demonstrating that visual features maximally contribute to ecological food choices (see Levy \& Glimcher, 2011; Foroni et al., 2013, 2016). Indeed, Kringelbach and Rolls (2004) argued that OFC neurons respond more strongly to the visual modality of foods, while Simmons, Martin, and Barsalou (2005) found that food pictures, similarly to real food, activate areas of the primary gustatory cortex (right insula) and areas implicated in reward such as the lateral OFC. On the other hand, a future extension of the present work would consider the use of real food choices would significantly modulate the accumulation of information patterns hereby found. Additionally, future studies should further control the possibility that endogenous processes despite those manipulated here may have influenced the across-trial variability in drift rate (e.g., attentional lapses; Steingroever, Wabersich, \& Wagenmakers, 2018). Another limitation is represented by the binary approach to the food choice. While it allows for simpler computations and faster decision processes - the latter particularly suited for the DDM approach - choices are rarely binary in the real world. We hope that our study shows that it is possible to study food choice with computational methods and that it can be extended to multiple items at the same time, as other authors have done (Helfer \& Shultz, 2014; Krajbich et al., 2015).

To conclude, we showed that food choices are complex decision processes that require the assessment of conflicting information, including one's hunger state, the calories of the food item and the subjective preference for those items. Furthermore, by capitalizing on the HDDM approach, this work sheds light on how nuanced computation models represent a promising new approach in understanding multifaceted decision-making and value assessment processes, such as those regarding food. Given the complexity of appetite, we believe that this approach can clarify the contribution of factors not considered in this study, such as socio-economical ones, food attitudes, pathological behaviors towards food (e.g., obesity and anorexia) and sensory features of foods.

\section{Conflicts of interest}

The authors declare no conflict of interest.

\section{Acknowledgements}

The authors wish to thank Carol Coricelli and Nora Chen for revising and providing precious feedback on a previous version of the paper.

\section{Appendix A. Supplementary data}

Supplementary data to this article can be found online at https:// doi.org/10.1016/j.appet.2019.03.009.

\section{References}

Bielser, M.-L., Cr'ez'e, C., Murray, M. M., \& Toepel, U. (2016). Does my brain want what my eyes like?-how food liking and choice influence spatio- temporal brain dynamics of food viewing. Brain and Cognition, 110, 64-73.

Brunstrom, J. M., \& Rogers, P. J. (2009). How many calories are on our plate? Expected fullness, not liking, determines meal-size selection. Obesity, 17(10), 1884-1890.

Buysse, D. J., Reynolds, C. F., Monk, T. H., Berman, S. R., \& Kupfer, D. J. (1989). The pittsburgh sleep quality index: A new instrument for psychiatric practice and research. Psychiatry Research, 28(2), 193-213.

Camerer, C. F., Loewenstein, G., \& Rabin, M. (2011). Advances in behavioral economics. Princeton University Press.

Cavanagh, J. F., Wiecki, T. V., Cohen, M. X., Figueroa, C. M., Samanta, J., Sherman, S. J., et al. (2011). Subthalamic nucleus stimulation reverses mediofrontal influence over decision threshold. Nature Neuroscience, 14(11), 1462-1467.

Charbonnier, L., Van Der Laan, L. N., Viergever, M. A., \& Smeets, P. A. (2015). Functional mri of challenging food choices: Forced choice between equally liked high-and lowcalorie foods in the absence of hunger. PLoS One, 10(7) e0131727.

Coppin, G., Delplanque, S., Bernard, C., Cekic, S., Porcherot, C., Cayeux, I., et al. (2014). Choice both affects and reflects preferences. The Quarterly Journal of Experimental 
Psychology, 67(7), 1415-1427.

Croker, H., Whitaker, K., Cooke, L., \& Wardle, J. (2009). Do social norms affect intended food choice? Preventive Medicine, 49(2), 190-193.

Curcio, G., Tempesta, D., Scarlata, S., Marzano, C., Moroni, F., Rossini, P. M., et al. (2013). Validity of the Italian version of the pittsburgh sleep quality index (PSQI). Neurological Sciences, 34(4), 511-519.

Finlayson, G., King, N., \& Blundell, J. E. (2007). Is it possible to dissociate 'liking' and 'wanting' for foods in humans? A novel experimental procedure. Physiology \& Behavior, 90(1), 36-42.

Foroni, F., Pergola, G., Argiris, G., \& Rumiati, R. I. (2013). The foodcast research image database (FRIDa). Frontiers in Human Neuroscience, 7.

Foroni, F., Pergola, G., \& Rumiati, R. I. (2016). Food color is in the eye of the beholder: The role of human trichromatic vision in food evaluation. Scientific Reports, 6, 37034.

Frank, S., Laharnar, N., Kullmann, S., Veit, R., Canova, C., Hegner, Y. L., et al. (2010). Processing of food pictures: Influence of hunger, gender and calorie content. Brain Research, 1350, 159-166.

Frey, D. (1986). Recent research on selective exposure to information. In L. Berkowitz (Vol. Ed.), Advances in experimental social psychology: Vol. 19, (pp. 41-80). New York: Academic Press.

Gelman, A., Carlin, J. B., Stern, H. S., \& Rubin, D. B. (2014). Bayesian data analysis, Vol. 2. Boca Raton, FL, USA: Chapman \& Hall/CRC.

Gelman, A., \& Rubin, D. B. (1992). Inference from iterative simulation using multiple sequences. Statistical Science, 457-472.

Gilhooly, C., Das, S. K., Golden, J., McCrory, M., Dallal, G., Saltzman, E., et al. (2007) Food cravings and energy regulation: The characteristics of craved foods and their relationship with eating behaviors and weight change during 6 months of dietary energy restriction. International Journal of Obesity, 31(12), 1849-1858.

Griffioen-Roose, S., Smeets, P. A., van den Heuvel, E., Boesveldt, S., Finlayson, G., \& de Graaf, C. (2014). Human protein status modulates brain reward responses to food cues. American Journal of Clinical Nutrition, 100(1), 113-122.

Helfer, P., \& Shultz, T. R. (2014). The effects of nutrition labeling on consumer food choice: A psychological experiment and computational model. Annals of the New York Academy of Sciences, 1331(1), 174-185.

Hoefling, A., \& Strack, F. (2010). Hunger induced changes in food choice. when beggars cannot be choosers even if they are allowed to choose. Appetite, 54(3), 603-606.

Kahnt, T., Park, S. Q., Haynes, J.-D., \& Tobler, P. N. (2014). Disentangling neural representations of value and salience in the human brain. Proceedings of the National Academy of Sciences, 111(13), 5000-5005.

Killgore, W. D., Young, A. D., Femia, L. A., Bogorodzki, P., Rogowska, J., \& YurgelunTodd, D. A. (2003). Cortical and limbic activation during viewing of high-versus lowcalorie foods. NeuroImage, 19(4), 1381-1394.

Koster, E. P. (2003). The psychology of food choice: Some often encountered fallacies. Food Quality and Preference, 14(5), 359-373.

Krajbich, I., Armel, C., \& Rangel, A. (2010). Visual fixations and the computation and comparison of value in simple choice. Nature Neuroscience, 13(10), 1292-1298.

Krajbich, I., Hare, T., Bartling, B., Morishima, Y., \& Fehr, E. (2015). A common mechanism underlying food choice and social decisions. PLoS Computational Biology, 11(10) e1004371.

Kringelbach, M. L., \& Rolls, E. T. (2004). The functional neuroanatomy of the human orbitofrontal cortex: Evidence from neuroimaging and neuropsychology. Progress in Neurobiology, 72(5), 341-372.

Lee, M. D., \& Wagenmakers, E.-J. (2014). Bayesian cognitive modeling: A practical course Cambridge University Press.

Levy, D. J., \& Glimcher, P. W. (2011). Comparing apples and oranges: Using rewardspecific and reward-general subjective value representation in the brain. Journal of Neuroscience, 31(41), 14693-14707.

Lieberman, D. (2014). The story of the human body: Evolution, health, and disease. Vintage Books.

Lindeman, M., \& Stark, K. (1999). Pleasure, pursuit of health or negotiation of identity? Personality correlates of food choice motives among young and middle-aged women. Appetite, 33(1), 141-161.

Mela, D. J. (2001). Determinants of food choice: Relationships with obesity and weight control. Obesity, 9(S11).

Menon, V., \& Uddin, L. Q. (2010). Saliency, switching, attention and control: A network model of insula function. Brain Structure and Function, 214(5-6), 655-667.

Mitchell, D. G. (2011). The nexus between decision making and emotion regulation: A review of convergent neurocognitive substrates. Behavioural Brain Research, 217(1), $215-231$.

Mormann, M. M., Koch, C., \& Rangel, A. (2011). Consumers can make decisions in as little as a third of a second. Judgment and Decision Making, 6(6), 520-530.

Mormann, M. M., Malmaud, J., Huth, A., Koch, C., \& Rangel, A. (2010). The drift diffusion model can account for the accuracy and reaction time of value-based choices under high and low time pressure. Udgment and Decision Making, 5(6), 437-449.

Ohla, K., Toepel, U., Le Coutre, J., \& Hudry, J. (2012). Visual-gustatory interaction: Orbitofrontal and insular cortices mediate the effect of high-calorie visual food cues on taste pleasantness. PLoS One, 7(3), e32434.

Padoa-Schioppa, C., \& Assad, J. A. (2006). Neurons in the orbitofrontal cortex encode economic value. Nature, 441(7090), 223-226.

Peirce, J. W. (2007). Psychopy-psychophysics software in python. Journal of Neuroscience Methods, 162(1), 8-13.

di Pellegrino, G., Magarelli, S., \& Mengarelli, F. (2010). Food pleasantness affects visual selective attention. The Quarterly Journal of Experimental Psychology, 64(3), 560-571.

Pergola, G., Foroni, F., Mengotti, P., Argiris, G., \& Rumiati, R. I. (2017). A neural signature of food semantics is associated with body-mass index. Biological Psychology, 129, 282-292.

Piech, R. M., Pastorino, M. T., \& Zald, D. H. (2010). All I saw was the cake. Hunger effects on attentional capture by visual food cues. Appetite, 54(3), 579-582.

Polivy, J., Herman, C. P., \& Howard, K. I. (1988). The restraint scale: Assessment of dieting. Dictionary of behavioral assessment techniques.

Pontzer, H., Brown, M. H., Raichlen, D. A., Dunsworth, H., Hare, B., Walker, K., et al. (2016). Metabolic acceleration and the evolution of human brain size and life history. Nature, 533(7603), 390-392.

Rangel, A. (2013). Regulation of dietary choice by the decision-making circuitry. Nature Neuroscience, 16(12), 1717-1724.

Ratcliff, R. (1978). A theory of memory retrieval. Psychological Review, 85(2), 59.

Ratcliff, R., \& Tuerlinckx, F. (2002). Estimating parameters of the diffusion model: Approaches to dealing with contaminant reaction times and parameter variability. Psychonomic Bulletin \& Review, 9, 438-481. https://doi.org/10.3758/BF03196302.

Read, D., \& Van Leeuwen, B. (1998). Predicting hunger: The effects of appetite and delay on choice. Organizational Behavior and Human Decision Processes, 76(2), 189-205.

Regenbogen, C., Johansson, E., Andersson, P., Olsson, M. J., \& Lundstrom, J. N. (2016). Bayesian-based integration of multisensory naturalistic perithreshold stimuli. Neuropsychologia, 88, 123-130.

Rohe, T., \& Noppeney, U. (2015). Cortical hierarchies perform bayesian causal inference in multisensory perception. PLoS Biology, 13(2) e1002073.

Shepherd, R. (2001). Does taste determine consumption? Understanding the psychology of food choice. Food, people and society (pp. 117-130). Springer.

Shepherd, R., \& Raats, M. (2006). The psychology of food choice, Vol. 3. Cabi.

Shepherd, R., \& Sparks, P. (1994). Modelling food choice. Measurement of food preferences (pp. 202-226). Springer.

Siep, N., Roefs, A., Roebroeck, A., Havermans, R., Bonte, M. L., \& Jansen, A. (2009). Hunger is the best spice: An fmri study of the effects of attention, hunger and calorie content on food reward processing in the amygdala and orbitofrontal cortex. Behavioural Brain Research, 198(1), 149-158.

Simmons, W. K., Martin, A., \& Barsalou, L. W. (2005). Pictures of appetizing foods activate gustatory cortices for taste and reward. Cerebral Cortex, 15(10), 1602-1608.

Spence, C., Okajima, K., Cheok, A. D., Petit, O., \& Michel, C. (2016). Eating with our eyes: From visual hunger to digital satiation. Brain and Cognition, 110, 53-63.

Spiegelhalter, D. J., Best, N. G., Carlin, B. P., \& Van Der Linde, A. (2002). Bayesian measures of model complexity and fit. Journal of the Royal Statistical Society: Series B, 64(4), 583-639.

Steingroever, H., Wabersich, D., \& Wagenmakers, E. J. (2018). Modeling across-trial variability in the wald drift rate parameter. Preprint retrieved at https://psyarxiv. com/7rspg.

Stroebele, N., \& De Castro, J. M. (2004). Effect of ambience on food intake and food choice. Nutrition, 20(9), 821-838.

Toepel, U., Knebel, J.-F., Hudry, J., le Coutre, J., \& Murray, M. M. (2009). The brain tracks the energetic value in food images. Neurolmage, 44(3), 967-974.

Towal, R. B., Mormann, M., \& Koch, C. (2013). Simultaneous modeling of visual saliency and value computation improves predictions of economic choice. Proceedings of the National Academy of Sciences, 110(40), E3858-E3867.

Vandekerckhove, J., Tuerlinckx, F., \& Lee, M. D. (2011). Hierarchical diffusion models for two-choice response times. Psychological Methods, 16(1), 44.

Van der Laan, L. N., De Ridder, D. T., Viergever, M. A., \& Smeets, P. A. (2012) Appearance matters: Neural correlates of food choice and packaging aesthetics. PLoS One, 7(7), e41738.

Wagenmakers, E. J., Van Der Maas, H. L., \& Grasman, R. P. (2007). An EZ-diffusion model for response time and accuracy. Psychonomic Bulletin \& Review, 14(1), 3-22.

Wiecki, T. V., Sofer, I., \& Frank, M. J. (2013). Hddm: Hierarchical bayesian estimation of the drift-diffusion model in python. Frontiers in Neuroinformatics, 7(August), 1-10.

Wrangham, R. (2009). Catching fire: How cooking made us human. Basic Books.

Zellner, D. A., Saito, S., \& Gonzalez, J. (2007). The effect of stress on men's food selection. Appetite, 49(3), 696-699. 